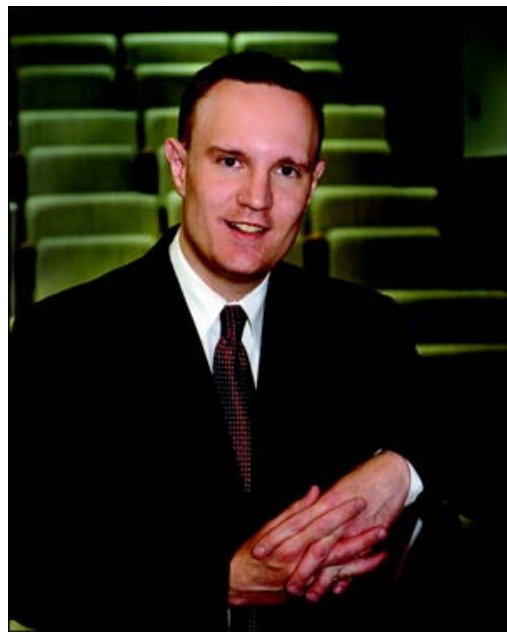

Bradley E. Karlin, PhD

\section{Bridging the gap in delivery of psychological treatments for posttraumatic stress disorder}

Scientific understanding of and treatment for posttraumatic stress disorder (PTSD) have come a long way in the past few decades. Not long ago, the diagnostic features and pathophysiology of what we now know as PTSD and previously knew as "shell shock" or "battle fatigue" were poorly understood and treatment was widely considered to be futile. Advancements in our understanding of the development, onset, classification, and treatment of PTSD over the last several decades now offer great promise to Veterans with PTSD returning from the conflicts in Iraq and Afghanistan, as well as to those who returned from conflict decades ago and non-Veteran trauma survivors. As the articles in this special issue of the Journal of Rehabilitation Research and Development illustrate, the current state of the science of and treatment for PTSD provide real and unprecedented opportunities for recovery for many individuals with PTSD.

Among the significant advancements in PTSD research is the development of specialized treatments for PTSD, including specific trauma-focused psychotherapies, such as prolonged exposure therapy (PE) and cognitive processing therapy (CPT). Rigorous research, including randomized controlled efficacy trials and effectiveness research, have shown that PE and CPT are quite effective in similar degree [1-5] and often yield outcomes that are enduring [6]. Much of this research has focused on non-Veteran sexual trauma survivors, though a recent spate of research has demonstrated the efficacy and effectiveness of these therapies with Veteran samples [3,5,7-10]. Based on the scientific evidence, CPT and PE are recommended in the Department of Veterans Affairs (VA)/Department of Defense (DOD) VA/ DOD Clinical Practice Guideline for Management of Post-Traumatic Stress for PTSD at the highest level, indicating "a strong recommendation that the intervention is always indicated and acceptable.”

Although the research evidence would suggest that trauma-focused psychotherapies should be considered a clinical mainstay for the treatment of PTSD, the clinical reality is that these and other evidence-based psychotherapies (EBPs) have largely languished in the laboratory for years and not made their way into therapy rooms in either private or public mental health care systems [11-12]. One survey of mental health professionals conducted in 2001 assessing the extent to which mental health providers provided evidence-based care for PTSD found that fewer than 10 percent of both generalist mental health providers and specialty PTSD mental health providers reported providing manualized psychotherapy for PTSD [12]. Among the key reasons identified in the literature for this science-to-practice gap are limited systematic training of providers in these therapies and lack of organizational support and infrastructures (e.g., the availability of 60-90 minute 
weekly sessions as these therapies require) to implement these treatments.

As an integrated healthcare system that establishes its own policies and services, the Veterans Health Administration (VHA) supports PE and CPT as first-line treatments for PTSD when clinically indicated. In fact, national VHA policy requires that all Veterans with PTSD have access to CPT or PE as designed and shown to be effective. To promote the availability of these treatments, VA has been working over the last several years to nationally disseminate and implement these therapies as part of a larger initiative to transform the VA mental health care delivery system [13]. This transformation process has led to both structural and process enhancements to mental health care delivery in VHA and to the addition of more than 7,000 staff to the mental health care workforce since 2005, bringing the current total to more than 20,000 staff.

As part of its efforts to nationally disseminate PE, CPT, and other EBPs, VA has established national competency-based staff training programs in these and other psychotherapies. As of April 1, 2012, VA has provided training in the delivery of CPT or PE to more than 4,300 mental health staff, with many of these staff receiving training in both therapies. Initial program evaluation results indicate that the training in and implementation of these therapies have resulted in significant and positive therapist, patient, and system outcomes [14]. Furthermore, as has been reported in the literature, many patients treated for PTSD with PE or CPT in VHA have also shown significantly reduced depression symptoms, even though depression is not a focus of either of these treatments. This positive "by-product" of these treatments is quite intriguing and may reflect overlap in diagnostic constructs and/or the inter-relationship between PTSD and depression symptoms.

In addition to the development of competencybased training in PE and CPT, VA has developed a variety of other mechanisms, including the placement of a Local EBP Coordinator at each medical center and a PTSD Mentor within each VHA region, to promote local clinical infrastructures that enable the full implementation and sustainability of these intensive therapies. All VA medical centers now provide PE or CPT, and virtually all facilities provide both therapies.

As stated in the VA/DOD Clinical Practice Guideline (CPG), it is essential that patient preference be considered before initiating a course of PE or CPT, or any PTSD treatment for that matter. Many VHA facilities have implemented pretherapy processes, such as orientation groups and other processes, to allow Veterans to make well-informed choices about the treatments they engage in. During such a pretherapy encounter, the Veteran is provided with information about $\mathrm{PE}, \mathrm{CPT}$, and/or other treatments; the efficacy of the treatment(s); and the potential utility of the treatment(s) for the Veteran's particular problems. Ultimately, the selection of treatment is made by the Veteran, in collaboration with the therapist before the initiation of care.

Significantly, PTSD symptoms are only one part of the clinical picture for many patients with PTSD. Sleep disturbance and pain, for example, are not infrequent psychological bedfellows of PTSD, yet they are often not systematically addressed in clinical practice; and, until recently, little ink was devoted to these conditions in the PTSD treatment research literature. In recent years, increasing research has addressed the assessment and management of sleep disturbance and pain within the context of PTSD care. Scientific advances in this area are critical to closing important loops in PTSD treatment and to providing comprehensive care to patients with PTSD. The current version of the VA/DOD CPG importantly recommends that sleep disturbance and pain be assessed in all patients with PTSD and provides treatment recommendations for these relatively common concomitant conditions. While some patients experience notable improvement in these symptoms (particularly when mild to moderate in nature) following a course of trauma-focused psychotherapy, others fail to reap such benefits. Additional research is needed to identify why some patients receive such secondary benefits from psychological treatments for PTSD and others do not.

Cognitive-behavioral therapy for insomnia (CBTI) is a highly effective treatment for insomnia that may yield significant benefit to many individuals 
with PTSD. The outcomes and effect sizes (ESs) from the clinical trials on CBT-I render it one of the most effective psychological treatments in existence. Further, CBT-I has been shown in research trials to be effective in individuals with PTSD, depression, and pain. In light of the very strong research evidence, CBT-I is recognized as a first-line treatment for insomnia by the National Institutes of Health Consensus Statement, Academy of Sleep Medicine, and British Association of Psychopharmacology. Moreover, its brief length (approximately 4-6 sessions) make CBT-I quite conducive to implementation in a variety of clinical settings; yet, CBT-I has been very infrequently available in clinical settings, in large part because of a shortage in trained providers. This large discrepancy between the effectiveness and efficiency of CBT-I on the one hand and its limited availability on the other was the focus of an article in the Journal of the American Medical Association entitled, "Despite effectiveness, behavioral therapy for chronic insomnia still underused" [15]. VA is working to nationally disseminate CBT-I and has established a competency-based training program to train staff in CBT-I. The VA CBT-I protocol and training materials were adapted to include specific content on implementing CBT-I with patients who have comorbid PTSD [16]. Preliminary program evaluation data from the VA CBT-I Training Program indicate substantial improvements among Veteran patients and large ESs on measures of insomnia severity (ES = $2.5)$ and physical quality of life $(E S=1.0)$ for Veterans who completed CBT-I $(n=64)$.

Imagery rehearsal therapy (IRT), a cognitivebehavioral therapy designed specifically for nightmares in individuals with PTSD, has shown promise. IRT has a Grade $\mathrm{C}$ recommendation in the VA/ DOD CPG. Additional research may help to further establish IRT as a primary treatment for PTSDrelated nightmares.

One major area of opportunity and focus for expanding and fully realizing the potential of evidence-based psychological treatments for PTSD is the delivery of such treatments through telehealth modalities. In fact, the delivery of trauma-focused psychotherapies and other treatments for PTSD and other mental disorders by using telehealth modali- ties will likely be the next frontier in evidence-based mental health care. VHA sees significant potential to further the reach of PE, CPT, and other evidencebased psychotherapies to a greater number of Veterans residing in rural and frontier communities by the delivery of these therapies through clinical video teleconferencing. This vision is supported by recent research reflected in the VA/DOD CPG, including research conducted within VHA, that has shown these therapies to be effective and well accepted by patients when delivered utilizing telehealth technologies, with results comparable to face-to-face delivery of these treatments. VHA has developed and is actively implementing a strategic plan to promote the delivery of PE and CPT telemental health services nationally. In addition, VHA has developed an EBP for PTSD tool kit with information on the technical and logistical requirements and clinical procedures for delivering CPT and PE through telemental health modalities that will be available to front-line clinicians and program leaders.

Clinical science and health technology provide unprecedented opportunities to effectively treat PTSD and transcend geographical and other access barriers. At the same time, the recent conflicts in Iraq and Afghanistan have helped further place PTSD into the national lexicon and have promoted public, political, and professional attention to the condition and to mental health more broadly. The time is now to bridge clinical science, practice, and technological innovations so that increasing numbers of Veterans and others with PTSD can reclaim their lives.

\section{Bradley E. Karlin, PhD}

VA Central Office, Office of Mental Health Services, Washington, DC

Email: Bradley.Karlin2@va.gov

\section{REFERENCES}

1. Chard KM. An evaluation of cognitive processing therapy for the treatment of posttraumatic stress disorder related to childhood sexual abuse. J Consult 
Clin Psychol. 2005;73(5):965-71. [PMID:16287396] http://dx.doi.org/10.1037/0022-006X.73.5.965

2. Foa EB, Hembree EA, Cahill SP, Rauch SA, Riggs DS, Feeny NC, Yadin E. Randomized trial of prolonged exposure for posttraumatic stress disorder with and without cognitive restructuring: outcome at academic and community clinics. J Consult Clin Psychol. 2005;73(5):953-64. [PMID:16287395] http://dx.doi.org/10.1037/0022-006X.73.5.953

3. Monson CM, Schnurr PP, Resick PA, Friedman MJ, Young-Xu Y, Stevens SP. Cognitive processing therapy for veterans with military-related posttraumatic stress disorder. J Consult Clin Psychol. 2006; 74(5):898-907. [PMID:17032094]

http://dx.doi.org/10.1037/0022-006X.74.5.898

4. Resick PA, Galovski TE, O’Brien Uhlmansiek M, Scher CD, Clum GA, Young-Xu Y. A randomized clinical trial to dismantle components of cognitive processing therapy for posttraumatic stress disorder in female victims of interpersonal violence. J Consult Clin Psychol. 2008;76(2):243-58. [PMID:18377121] http://dx.doi.org/10.1037/0022-006X.76.2.243

5. Schnurr PP, Friedman MJ, Engel CC, Foa EB, Shea MT, Chow BK, Resick PA, Thurston V, Orsillo SM, Haug R, Turner C, Bernardy N. Cognitive behavioral therapy for posttraumatic stress disorder in women: a randomized controlled trial. JAMA. 2007;297(8): 820-30. [PMID:17327524]

http://dx.doi.org/10.1001/jama.297.8.820

6. Resick PA, Williams LF, Suvak MK, Monson CM, Gradus JL. Long-term outcomes of cognitive-behavioral treatments for posttraumatic stress disorder among female rape survivors. J Consult Clin Psychol. 2012;80(2):201-10. [PMID:22182261]

http://dx.doi.org/10.1037/a0026602

7. Chard KM, Schumm JA, Owens GP, Cottingham SM. A comparison of OEF and OIF veterans and Vietnam veterans receiving cognitive processing therapy. J Trauma Stress. 2010;23(1):25-32. [PMID:20146255]

8. Nacasch N, Foa EB, Fostick L, Polliack M, Dinstein Y, Tzur D, Levy P, Zohar J. Prolonged exposure therapy for chronic combat-related PTSD: a case report of five veterans. CNS Spectr. 2007;12(9):690-95.

[PMID:17805215]

9. Rauch SA, Defever E, Favorite T, Duroe A, Garrity C, Martis B, Liberzon I. Prolonged exposure for PTSD in a Veterans Health Administration PTSD clinic. J Trauma Stress. 2009;22(1):60-64.

\section{[PMID:19145643]}

http://dx.doi.org/10.1002/jts.20380

10. Tuerk PW, Yoder M, Ruggiero KJ, Gros DF, Acierno R. A pilot study of prolonged exposure therapy for posttraumatic stress disorder delivered via telehealth technology. J Trauma Stress. 2010;23(1):116-23. [PMID:20135675]

11. Goisman RM, Warshaw MG, Keller MB. Psychosocial treatment prescriptions for generalized anxiety disorder, panic disorder, and social phobia, 19911996. Am J Psychiatry. 1999;156(11):1819-21. [PMID:10553751]

12. Rosen CS, Chow HC, Finney JF, Greenbaum MA, Moos RH, Sheikh JI, Yesavage JA. VA practice patterns and practice guidelines for treating posttraumatic stress disorder. J Trauma Stress. 2004;17(3): 213-22. [PMID:15253093] http://dx.doi.org/10.1023/ B:JOTS.0000029264.23878.53

13. Edwards DJ. Transforming the VA. Behav Healthc. 2008;28(7):14-17. [PMID:18754557]

14. Karlin BE, Ruzek JI, Chard KM, Eftekhari A, Monson CM, Hembree EA, Resick PA, Foa EB. Dissemination of evidence-based psychological treatments for posttraumatic stress disorder in the Veterans Health Administration. J Trauma Stress. 2010;23(6): 663-73. [PMID:21171126] http://dx.doi.org/10.1002/jts.20588

15. Lamberg L. Despite effectiveness, behavioral therapy for chronic insomnia still underused. JAMA. 2008; 300(21):2474-75. [PMID:19050184] http://dx.doi.org/10.1001/jama.2008.719

16. Manber R, Carney C, Edinger J, Epstein D, Friedman L, Haynes PL, Karlin BE, Pigeon W, Siebern AT, Trockel M. Dissemination of CBTI to the nonsleep specialist: protocol development and training issues. J Clin Sleep Med. 2012;8(2):209-18. [PMID:22505869]

This article and any supplementary material should be cited as follows:

Karlin BE. Bridging the gap in delivery of psychological treatments for posttraumatic stress disorder. J Rehabil Res Dev. 2012;49(5):xiii-xvi. http://dx.doi.org/10.1682/JRRD.2012.01.0006

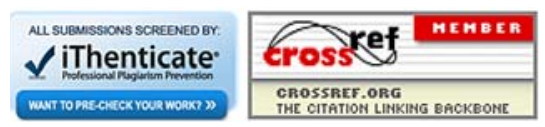

